\title{
Normal human dermis contains distinct populations of CD11C+BDCA-1+ dendritic cells and $\mathrm{CD}_{163}{ }^{+} \mathrm{FXIIIA}{ }^{+}$macrophages
}

\author{
Lisa C. Zaba, ${ }^{1}$ Judilyn Fuentes-Duculan, ${ }^{1}$ Ralph M. Steinman, ${ }^{2}$ \\ James G. Krueger, ${ }^{1}$ and Michelle A. Lowes ${ }^{1}$
}

${ }^{1}$ Laboratory for Investigative Dermatology and 'Laboratory of Cellular Physiology and Immunology, The Rockefeller University, New York, New York, USA.

\begin{abstract}
We used a panel of monoclonal antibodies to characterize DCs in the dermis of normal human skin. Staining for the CD11c integrin, which is abundant on many kinds of DCs, revealed cells in the upper dermis. These cells were positive for blood DC antigen-1 (BDCA-1; also known as CD1c), HLA-DR, and CD45, markers that are also expressed by circulating myeloid DCs. A small subset of CD11 ${ }^{+}$dermal cells expressed DEC-205/ CD205 and DC-lysosomal-associated membrane glycoprotein/CD208 (DC-LAMP/CD208), suggesting some differentiation or maturation. When BDCA-1 ${ }^{+}$cells were selected from collagenase digests of normal dermis, they proved to be strong stimulators for $T$ cells in a mixed leukocyte reaction. A second major population of cells located throughout the dermis was positive for factor XIIIA (FXIIIA), but lacked CD11c and BDCA-1. They expressed the macrophage scavenger receptor CD163 and stained weakly for HLA-DR and CD45. Isolated $\mathrm{CD}_{163}{ }^{+}$dermal cells were inactive in stimulating $\mathrm{T}$ cell proliferation, but in biopsies of tattoos, these cells were selectively laden with granular pigments. Plasmacytoid DCs were also present in the dermis, marked by CD123 and BDCA-2. In summary, the normal dermis contains typical immunostimulatory myeloid DCs identified by CD11c and BDCA-1, as well as an additional population of poorly stimulatory macrophages marked by CD163 and FXIIIA.
\end{abstract}

\section{Introduction}

DCs represent a major resident leukocyte population in human skin. Two main types of DCs are found in noninflamed skin: epidermal Langerhans cells (LCs) and dermal DCs $(1,2)$. LCs express Langerin/CD207, an endocytic receptor that localizes to and forms Birbeck granules, as well as the CD1a class I-like molecule that presents glycolipids (3). Dermal DCs have long been defined on the basis of expression of a clotting factor, the transglutaminase factor XIIIA (FXIIIA) (4). Studies that define dermal DCs as FXIIIA ${ }^{+}$ have often relied on fluorescence-activated cell sorting (FACS) analysis and functional studies using bulk tissue or enzymatically manipulated émigrés that "crawl out" of the dermis over a variable incubation period, which may increase FXIIIA expression $(5,6)$.

Currently, so-called myeloid or conventional DCs in many tissues are often identified on the basis of high expression of HLA-DR antigen-presenting molecules and the CD11c integrin. DCs are negative for Lin, a cocktail of antibodies to other cell lineages, including T cells (CD3), B cells (CD19 and CD20), monocytes (CD14), and granulocytes and NK cells (CD16 and CD56) $(7,8)$. Circulating myeloid DCs can be further classified as 3 mutually exclusive subsets - in order of immunostimulatory capacity, blood DC antigen-1-positive (BDCA- $1^{+}$), CD16 $6^{+}$, and $\mathrm{BDCA}-3^{+}(9)-$ but these markers have seen very little use in studies of the skin.

Because a large number of prevalent dermatologic conditions from atopic dermatitis to psoriasis are characterized by extensive

Nonstandard abbreviations used: BDCA, blood DC antigen; DC-LAMP, DC-lysosomal-associated membrane glycoprotein; DC-SIGN, DC-specific ICAM-3-grabbing nonintegrin; FACS, fluorescence-activated cell sorting; FXIIIA, factor XIIIA; LC, Langerhans cell; MFI, median fluorescence intensity; MLR, mixed leukocyte reaction; MMR, macrophage mannose receptor; PDC, plasmacytoid DC.

Conflict of interest: The authors have declared that no conflict of interest exists. Citation for this article: J. Clin. Invest. 117:2517-2525 (2007). doi:10.1172/JCI32282. dermal $\mathrm{T}$ cell infiltration, and as DCs are pivotal antigen-presenting cells for $\mathrm{T}$ cells, it is important to pursue these distinct phenotypic definitions of DCs in normal skin and peripheral blood. Here we report that CD11c and FXIIIA marked mutually exclusive populations, the former coexpressing BDCA-1 (also known as CD1c; ref. 10), and the latter uniformly positively for CD163, a scavenger receptor for hemoglobin/haptoglobin complexes. CD $11 \mathrm{c}^{+}$cells were more typical of DCs, with higher HLA-DR expression and T cell-stimulating activity. Surprisingly, FXIIIA ${ }^{+}$cells behaved more like macrophages since they were weak initiators of $\mathrm{T}$ cell responses in the mixed leukocyte reaction (MLR) and had numerous phagocytosed pigment-containing vacuoles in a tattoo.

\section{Results}

FXIIIA $A^{+}$and $C D 11 c^{+}$cells are discrete dermal populations. Immunohistochemistry of normal human dermis showed distinct staining patterns for FXIIIA ${ }^{+}$and CD $11 \mathrm{c}^{+}$cells (Figure 1A). The larger FXIIIA ${ }^{+}$cells were located throughout the dermis. In contrast, $\mathrm{CD} 11 \mathrm{c}^{+}$myeloid cells were located in the papillary and upper reticular dermis. We counted the absolute number

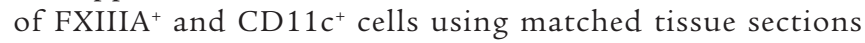
from 15 normal volunteers (Figure 1B). In the epidermis there

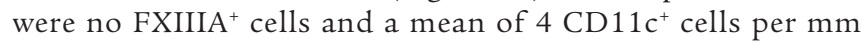
$(P=0.04)$. In the dermis, there was a mean of 83 FXIIIA $^{+}$cells

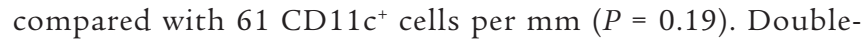
labeled immunofluorescence using FXIIIA and CD11c confirmed that these 2 antigens were not expressed on the same cell (Figure 1C). A polyclonal FXIIIA antibody has typically been used for staining dermal DCs in the past. We used FXIIIA affinity-purified sheep antibody for all of our double-label immunofluorescence studies, as we found it to yield the best 
A

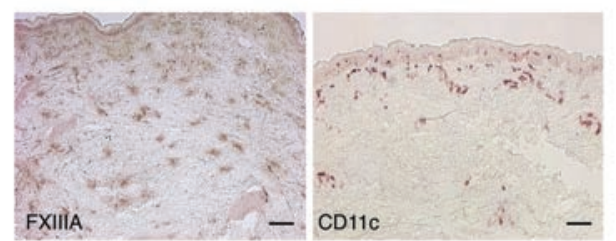

B

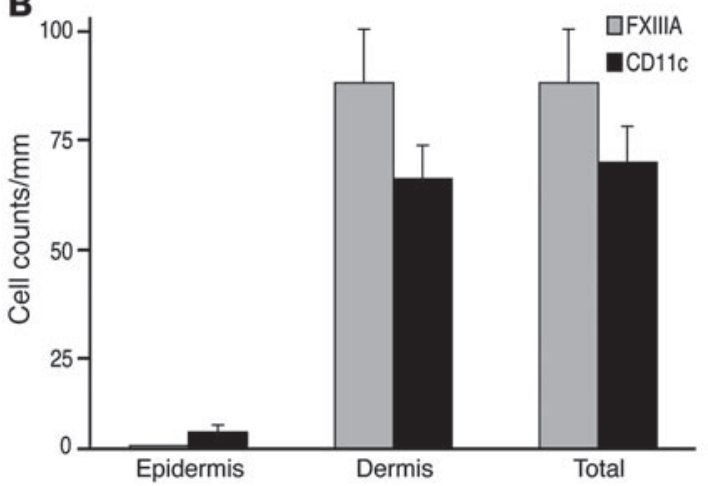

C
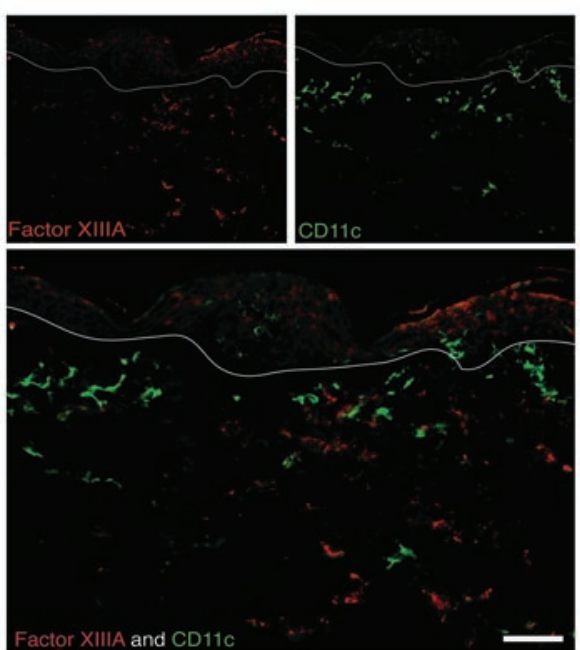

Figure 1

FXIIIA+ and CD11 ${ }^{+}$cells are unique dermal populations. (A) Immunohistochemistry on normal human skin using FXIIIA (left panel) and CD11C (right panel) antibodies $(n=15)$. FXIIIA+ cells were spread throughout the dermis, while CD11 $\mathrm{C}^{+}$cells were mainly localized to the superficial dermis. (B) There were similar numbers of $\mathrm{CD}_{11 \mathrm{C}^{+}}$and FXIIIA+ cells per mm in normal dermis. Error bars indicate SEM. (C) FXIIIA and CD11C identified 2 discrete populations. White lines denote dermo-epidermal junction. Scale bars: $100 \mu \mathrm{m}$.

staining. There was almost complete coexpression of the sheep FXIIIA antibody and a commonly used mouse monoclonal (clone AC-1A1), while other mouse monoclonal antibodies to FXIIIA produced weaker and less consistent staining (data not shown). These data indicate that 2 commonly used markers for dermal DCs are actually expressed by comparably abundant but different populations.

FXIIIA $^{+}$and CD $11 c^{+}$populations are not LCs or plasmacytoid DCs. To further characterize these 2 dermal cell populations, we first assessed expression of markers used to identify LCs and/or plasmacytoid DCs (PDCs), each applied in combination with antibodies to either FXIIIA or CD11c. Neither FXIIIA nor CD11c colocalized with the LC markers CD1a (Figure 2, A and B) and Langerin (Supplemental Figure 1, A and B; supplemental material available online with this article; doi:10.1172/JCI32282DS1), nor the PDC markers CD123 (Figure 2, C and D) and BDCA-2 (Supplemental Figure $1, \mathrm{C}$ and D). Therefore, FXIIIA ${ }^{+}$and CD $11 \mathrm{c}^{+}$cells are distinct from additional LC and PDC populations in normal skin. Table 1 summarizes the expression of various leukocyte markers on the FXIIIA ${ }^{+}$and CD $11 \mathrm{c}^{+}$cells.

CD11 $c^{+}$cells coexpress the blood DC marker BDCA-1, and a small fraction also express DC-lysosomal-associated membrane glycoprotein/ CD208 and DEC-205/CD205. BDCA-1 also marks a population of $\mathrm{CD} 11 \mathrm{c}^{+}$myeloid DCs in blood, and we noted coexpression of these 2 markers in the dermis as well (Figure 3, A and B). Two other markers that are expressed by tissue DCs are lysosomal marker DC-lysosomal-associated membrane glycoprotein/ CD208 (DC-LAMP/CD208) and endocytic receptor DEC-205/ CD205. A small fraction of the CD11 $\mathrm{c}^{+}$cells expressed these 2 markers (Figure 3, C-F). The fraction of CD11 $\mathrm{c}^{+} \mathrm{DC}-\mathrm{LAMP}^{+}$cells was qualitatively smaller than the fraction of CD $11 \mathrm{c}^{+} \mathrm{DEC}-205^{+}$ cells. DC-LAMP/CD208 is expressed during DC maturation, and we noted that cells positive for this marker were often in dermal aggregates with other CD $11 \mathrm{c}^{+} \mathrm{DC}-\mathrm{LAMP}^{-}$cells. These results indicate that $\mathrm{CD} 11 \mathrm{c}^{+}$cells in the dermis share features with the myeloid DCs in blood, but some are in a more mature state of differentiation (11).

FXIIIA $^{+}$cells express the macrophage marker and scavenger receptor CD163. CD163, a hemoglobin/haptoglobin complex scavenger receptor, identifies tissue resident macrophages, and it was the only marker we studied that was uniformly coexpressed by FXIIIA $^{+}$cells and not by CD $11 c^{+}$cells (Figure 4, A and B). Several other markers were expressed on a fraction of FXIIIA ${ }^{+}$and $\mathrm{CD} 11 \mathrm{c}^{+}$cells. These included the uptake receptors macrophage mannose receptor/CD206 (MMR/CD206; Figure 4, C and D), DC-specific ICAM-3-grabbing nonintegrin/CD209 (DC-SIGN/ CD209; Figure 4, E and F), CD45, and HLA-DR (Figure 5). In normal skin, both MMR/CD206 and DC-SIGN/CD209 were more abundant on macrophages (FXIIIA ${ }^{+}$cells) than DCs $\left(\right.$CD $11 c^{+}$ cells), which is consistent with recent studies in inflamed skin and lymph nodes $(11,12)$. Reciprocally, CD45 and HLA-DR were more abundant on CD $11 \mathrm{c}^{+}$than on FXIIIA ${ }^{+}$cells. All these observations are consistent with the interpretation that $\mathrm{CD} 11 \mathrm{c}^{+}$ cells in the dermis are part of the DC pathway of differentiation, while FXIIIA ${ }^{+}$cells are more macrophage-like.

Other markers of potential interest. Other markers used for characterization of FXIIIA ${ }^{+}$and CD11 $\mathrm{c}^{+}$cells were CD14 (identifies monocytes); CD68, CD11b, and RFD7 (commonly used macrophage markers); and CD63 (present on macrophages and DCs). These markers were not helpful in discriminating these 2 populations in normal skin (Supplemental Figure 2). CD14, a component of the LPS receptor on monocytes (13), was present on a few FXIIIA $^{+}$cells and CD $11 \mathrm{c}^{+}$cells. We have previously shown that CD68 identifies a subset of CD $11 c^{+}$cells that produce IL-20 in psoriasis (14), so this is not an exclusive macrophage marker. In keeping with this, CD68 also stained both CD11c ${ }^{+}$and FXIIIA 
A
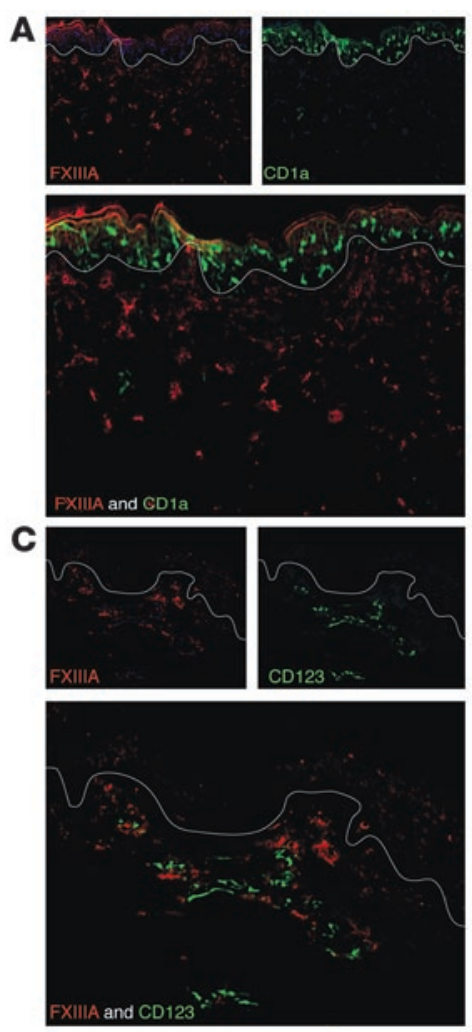
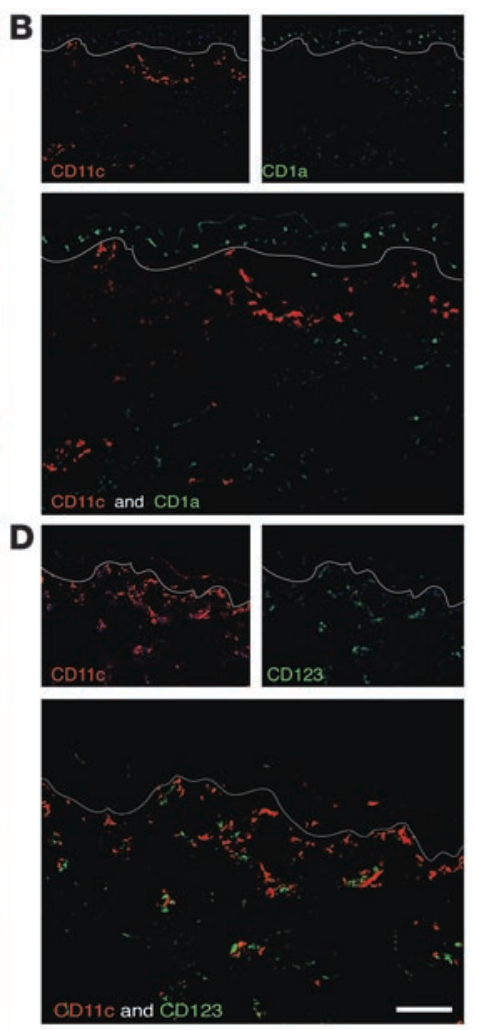

\section{Figure 2}

$\mathrm{FXIIIA}^{+}$and $\mathrm{CD} 11 \mathrm{C}^{+}$populations are not $\mathrm{LCs}$ or plasmacytoid DCs. Neither FXIIIA nor CD11c showed coexpression with Langerhans antigen CD1a (A and B) or plasmacytoid antigen CD123 (C and D). Scale bar: $100 \mathrm{~mm}$. cells. CD11b and RFD7, considered to be common macrophage markers $(13,15)$, were present on both $\mathrm{CD}_{11 \mathrm{c}^{+} \text {and FXIIIA }}^{+}$cells. CD63, an MHC class II internalization antigen, marked occasional $\mathrm{CD}_{11 \mathrm{c}^{+} \text {and FXIIIA }}{ }^{+}$cells in normal skin.

Isolated $C D 11 c^{+}$dermal cells contain nonoverlapping major and minor populations of $B D C A-1^{+}$and $B D C A-3^{+}$cells. To confirm our in situ data and assess whether the 3 myeloid DC subsets found in blood were also found in tissue, we performed 6-color FACS on single-cell suspensions of normal human skin compared with peripheral blood, using a custom-made group of monoclonal antibodies to lineage (Lin) that did not contain monoclonal antibodies to CD14 or CD16. Peripheral blood FACS dot plots are shown in Figure 6, A and B, and the parallel analysis from dermal cells is shown in Figure 6, C and D. There were 3 discrete Lin ${ }^{-}$ CD $11 c^{+} \mathrm{HLA}-\mathrm{DR}^{+} \mathrm{DC}$ populations in peripheral blood, BDCA- $1^{+}$, CD16 ${ }^{\text {hi }}$, and $\mathrm{BDCA}-3^{+}$(Figure $\left.6 \mathrm{~A}\right)$, but only 2 clear populations in skin, $\mathrm{BDCA}-1^{+} \mathrm{CD} 16^{\text {lo }}$ and $\mathrm{BDCA}-3^{+} \mathrm{CD} 16^{\text {lo }}$ (Figure $6 \mathrm{C}$ ). In blood, 2 discrete $\mathrm{CD} 11 \mathrm{c}^{+}$DC populations, BDCA- $1^{+}$and $\mathrm{BDCA}-3^{+}$, were both HLA-DR ${ }^{+} \mathrm{CD} 14^{-}$(Figure $6 \mathrm{~B}$ ). In contrast, BDCA-1+ cells from skin increased HLA-DR expression, and BDCA- $3^{+}$cells acquired low-level CD14 expression (Figure 6D). We also confirmed that BDCA-1 and BDCA-3 identified discrete populations in normal skin in situ (data not shown).

BDCA-1 and CD163 are superior markers to CD11c and FXIIIA, respectively, for FACS of isolated dermal cells. To begin to test the functional properties of cell populations isolated from dermis, we required markers that would be optimal for FACS. While culture conditions may alter cellular surface phenotype, it was important to compare DC populations using the new BDCA-1 and CD163 markers with previous studies on dermal DCs. It was necessary to balance obtaining sufficient cells to study with as little manipulation as possible. An overnight culture in dispase/collagenase media (16) yielded insufficient cells to perform our MLR experiments. Enzymic alteration of surface epitopes is also possible, although experiments on PBMCs did not show loss of FACS staining after culture in dispase collagenase

\section{Table 1}

Characterization of CD11C+ and FXIIIA+ cells in normal human dermis

\begin{tabular}{llcc} 
Antigen & Main cell location & FXIIIA & CD11c \\
CD1a & LCs, IDECs & - & - \\
Langerin/CD207 & LCs & - & - \\
CD123 & PDCs & - & - \\
BDCA-2 & PDCs & - & - \\
BDCA-1 & Myeloid DCs & - & ++ \\
DC-LAMP/CD208 & Mature DCs & - & + \\
DEC-205/CD205 & Mature DCs & - & + \\
CD163 & Macrophages & +++ & - \\
MMR/CD206 & Macrophages, DCs & ++ & ++ \\
DC-SIGN/CD209 & Macrophages, immature DCs & +++ & ++ \\
CD45 & Bone marrow-derived cells & + & +++ \\
HLA-DR & Antigen-presenting cells & + & ++ \\
CD14 & Monocyte-derived cells & + & + \\
CD68 & Macrophages, DCs & + & + \\
CD11b & Myeloid cells & + & ++ \\
RFD7 & Macrophages, DCs & ++ & + \\
CD63 & Macrophages, DCs & + & + \\
\hline
\end{tabular}

Qualitative coexpression of FXIIIA and CD11c with additional antibody is denoted as follows: - , no coexpression; +, some cells show coexpression; ++, a moderate number of cells show coexpression; +++, most cells show coexpression. IDEC, inflammatory dendritic epidermal cell. 

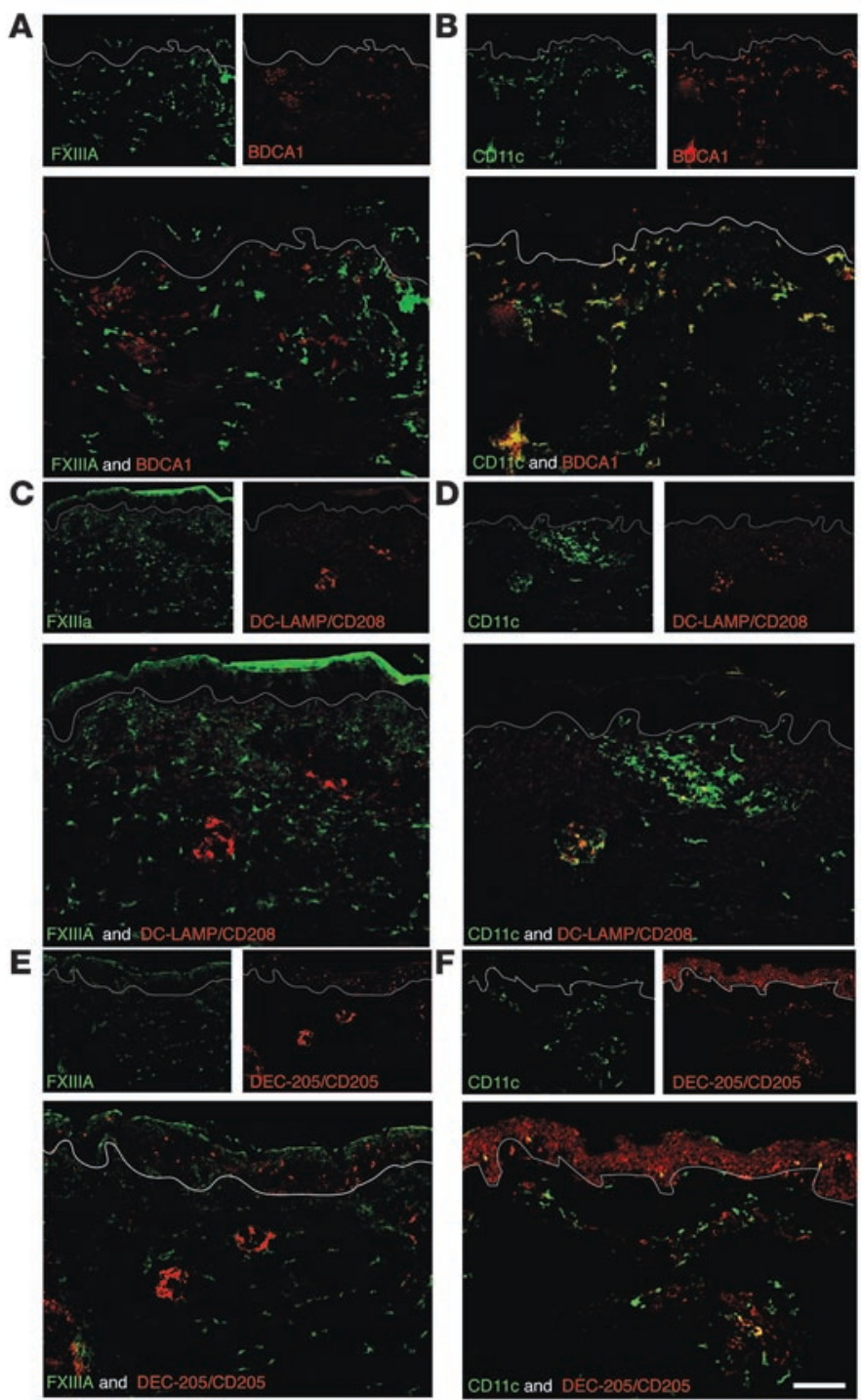

Figure 3

CD11C ${ }^{+}$cells are defined by BDCA-1, DC-LAMP/CD208, and DEC-205/ CD205. FXIIIA did not overlap with BDCA-1 (A), DC-LAMP/CD208 (C), or DEC-205/CD205 (E). Most CD11 ${ }^{+}$cells coexpressed BDCA-1 (B). Small subsets of CD11C cells coexpressed DC-LAMP/CD208 (D) and DEC-205/CD205 (F). Scale bar: $100 \mu \mathrm{m}$.

mix (data not shown). This method was modified to allow 1-2 days of culture so any lost surface markers could be reexpressed and to allow DCs and macrophages to move out of the scored upper and lower dermal surfaces. Comparison of BDCA-1 versus CD163 on the first day of culture (after overnight culture in dispase/collagenase) and after 24 and 48 hours of culture showed a similar clear distinction of the 2 populations (data not shown). Culture of bulk dermal cells from normal skin using this method with and without DC maturation cytokines (IL-1 $\beta$, IL-6, TNF, and PGE 2 ) showed that there was surprisingly little maturation of myeloid DCs $\left(\mathrm{BDCA}-1^{+}\right)$as measured by HLA-DR and CD83 (data not shown).

We found that BDCA-1, which colabeled more than $90 \%$ of

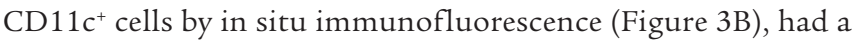

much higher median fluorescence intensity (MFI) than did CD11c (data not shown). Likewise, CD163, which showed $100 \%$ coexpression with FXIIIA in situ (Figure 4A), proved to be a much better FACS marker. FXIIIA antibodies bound nonspecifically to dermal cells in suspension, perhaps as a result of adherence of platelet fragments rich in FXIIIA, which is upregulated during cell culture $(5,6)$.

Double-label immunofluorescence of BDCA-1 and CD163 confirmed that they were distinct populations in situ (Figure 7A), which supports their use as alternative markers for CD11c and FXIIIA, respectively, in FACS studies. Bulk dermal single-cell suspensions showed distinct $\mathrm{BDCA}-1^{+}$and CD $163^{\text {hi }}$ populations by FACS (Figure 7B). BDCA- $1^{+}$gated cells had a higher MFI for the CD11c than the CD163 hi population (Figure 7C), most likely because of increased sensitivity of FACS compared with immunohistochemistry. CD $163^{\text {hi }}$ cells had a higher MFI for FXIIIA than did BDCA- $1^{+}$cells (Figure 7C). BDCA-1 $1^{+}$gated cells also had a higher MFI for HLA-DR and CD45 than did CD163 ${ }^{\text {hi }}$ gated cells (Figure 7C). A subset of BDCA- $1^{+}$cells was positive for DC maturation markers CD86, CD83, and DC-LAMP/CD208 (Figure 7D). Because $\mathrm{B}$ cells may also express BDCA-1, we confirmed that none of the BDCA $-1^{+}$cells were CD $19^{+}$by FACS (data not shown). Thus the dermal cells have a pattern of expression similar to in situ characterization.

$B D C A-1^{+}$cells are a major immunostimulatory population from normal buman skin. To test for the immunostimulatory properties of dermal leukocytes, we focused on FACS-sorted populations of $\mathrm{BDCA}-1^{+}$and $\mathrm{CD} 163^{\text {hi }}$ cells released from the dermis with collagenase. Data are summarized in Supplemental Table 3, and Figure 8 shows representative FACS plots of the MLR. The sorted cells were $99 \%$ pure compared with isotype (Figure $8 \mathrm{~A})$. In the MLR induced by in vitro monocytederived mature DCs, $63 \%$ of the surviving T cells had undergone extensive proliferation at a stimulator/responder ratio of $1: 100$ on day 8 after sorting (Figure $8 B$ ). In parallel cultures stimulated by BDCA- $1^{+}$cells, $9.1 \%$ of the T cells had proliferated (1:10 ratio), compared with $2.1 \%$ of $C D 163^{+}$cells and $1.0 \%$ background $\mathrm{T}$ cell proliferation (Figure $8 \mathrm{C}$ ). When these sorted $\mathrm{BDCA}-1^{+}$and $\mathrm{CD} 163^{+}$populations were cultured for 2 days in a DC-maturing cytokine cocktail before setting up the MLR, the immunostimulatory capability of BDCA- $1^{+}$cells was increased to $25.2 \%$ (1:100 ratio), but the capacity of $\mathrm{CD} 163^{+}$ cells was unchanged $(2.2 \% ; 1: 250$ ratio, low because of low cell survival during the culture period; Figure 8D). BDCA- $1^{+}$ sorted cells were cultured for 2 days without cytokines, and the supernatant from these cells after culture also increased $\mathrm{T}$ cell proliferation (data not shown).

$\mathrm{CD}_{163^{+}}$cells phagocytose large particles in a tattoo and have the structural features of macrophages. Ultrathin sections of a green dye tattoo were cut from tattoo-bearing normal skin. These sections confirmed that the dye was intracytoplasmic and located mostly in cells clustered around blood vessels (Figure 9A). Electron microscopy of the tattoo revealed membrane-bound (Figure 9B, blue arrow) tattoo dye particles (red arrow) and microvillous projections (green arrow), confirming the identity of these cells as macrophages. Immunohistochemistry using BDCA-1 (Figure 9C) and CD163 (Figure 9D) showed that dye-laden cells were CD163 ${ }^{+}$ and not $\mathrm{BDCA}-1^{+}$, confirming that typical macrophages rather than DCs had ingested the pigment. 

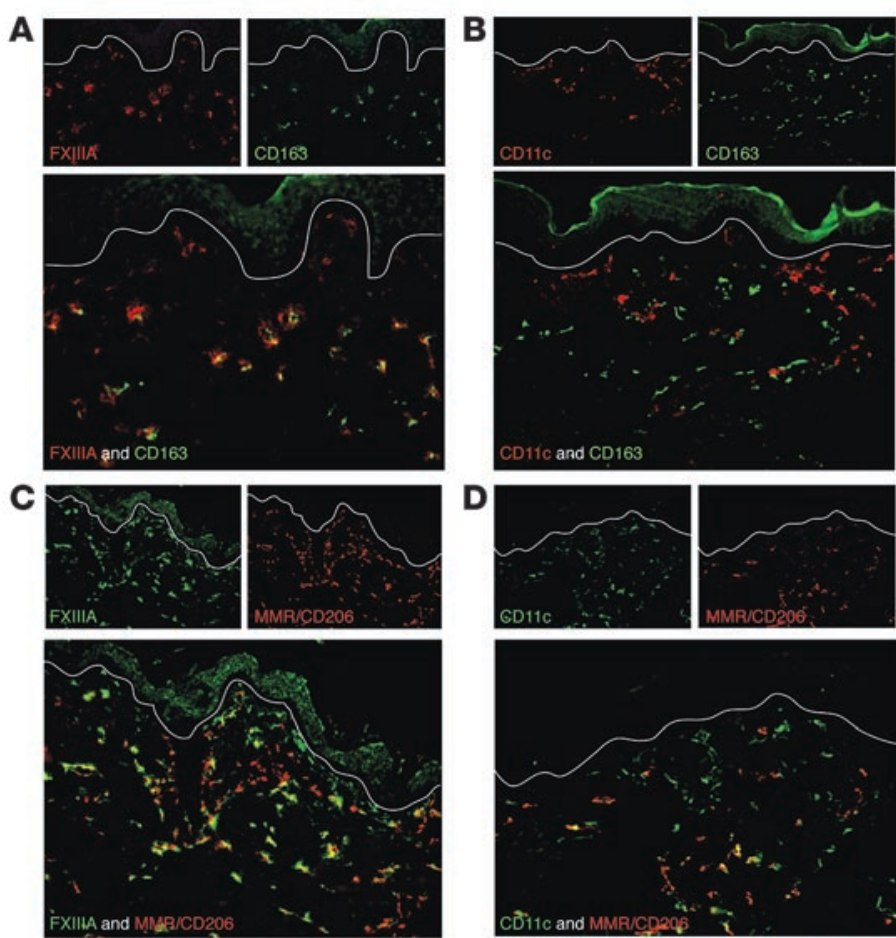

E
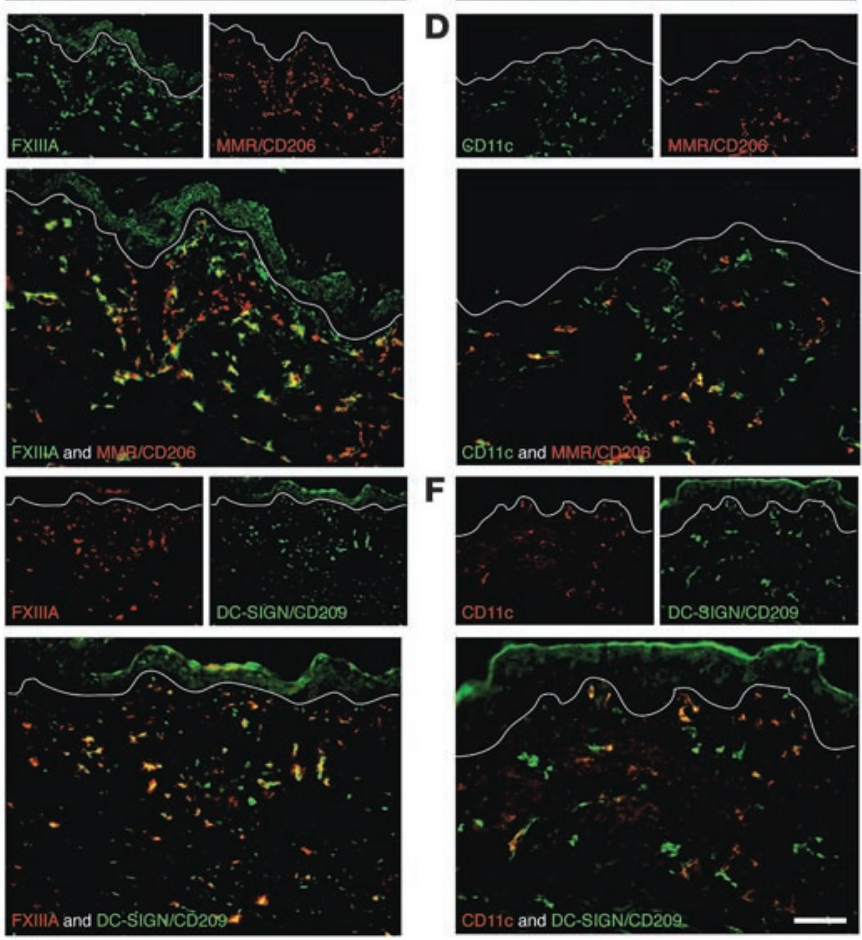

Figure 4

The macrophage marker CD163 defines $\mathrm{FXIIIA}^{+}$cells. FXIIIA+ cells expressed macrophage marker CD163 (A), and a subset overlapped with MMR/CD206 (C) and DC-SIGN/CD209 (E). CD11C did not overlap with CD163 (B), but a subset overlapped with MMR/CD206 (D) and DC-SIGN/ CD209 (F). Scale bar: $100 \mu \mathrm{m}$.

\section{Discussion}

DCs are important sentinels of the cutaneous immune system, performing central roles in both the innate and the acquired immune systems. Previous characterization of DC subsets in human dermis has been influenced by results with a rabbit polyclonal antibody to FXIIIA, which identifies dermal cells with a dendritic morphology (4, 17-20). Here we have characterized populations of cells within the normal dermis and found that, surprisingly, cells expressing the CD11c integrin and the BDCA-1 antigen-presenting molecule represent a distinct population functionally differentiated along the DC pathway, whereas FXIIIA ${ }^{+}$ cells are differentiated along the macrophage pathway. Whereas mouse skin contains approximately $1 \mathrm{CD} 11 \mathrm{c}^{+} \mathrm{DC}$ for every 5 macrophages (21), the concentration of CD $11 c^{+}$DCs in human skin is much higher, closer to a 1:1 ratio.
CD11c colocalized with several well-recognized DC markers: BDCA-1, DC-LAMP/CD208, and DEC-205/CD205. BDCA-1, also known as CD1c (10), is an invariant MHC class I-like antigen receptor molecule that recognizes lipids in mycobacterial cell walls. BDCA-1 is found on immature and mature DCs, and also on a subset of B cells. BDCA-1, in the absence of the B cell markers CD19 and CD20, would therefore seem to be a valuable marker to compare dermal DCs in both normal skin (22) and inflammatory skin diseases (23). DC-LAMP/ CD208 is a lysosomal protein that specifically marks mature DCs (24). DEC-205/CD205 is a surface receptor that participates in DC antigen endocytosis; its expression increases during maturation, and it has been previously demonstrated in normal human skin (25). The low frequency of CD11 $\mathrm{c}^{+} \mathrm{DCs}$ expressing these 2 antigens observed in normal skin is consistent with their expected immature DC status. CD11c cells also stained brightly with HLA-DR and CD45, confirming their antigen-presenting potential and bone marrow origin, respectively. The phenotype of $\mathrm{BDCA}-1^{+}$cells from skin indicates that they are all CD45 ${ }^{\text {hi HLA-DR }}{ }^{\text {hi }}$ and that a subset of these cells is $\mathrm{CD}^{\circ} 6^{+}$and $\mathrm{CD} 83^{+}$.

Our studies show that FXIIIA identifies a population of tissue-resident macrophages in normal skin. The only antibody that overlapped completely with FXIIIA in situ was the scavenger receptor CD163, which is selectively expressed on monocytes and macrophages (reviewed in ref. 26). The bestcharacterized function of CD163 is to bind hemoglobin/ haptoglobin complexes, which may be important in homeostasis. There was low-level CD45 and HLA-DR expression, consistent with other tissue macrophages, and limited antigen-presenting capacity (27). MMR/CD206 and DC-SIGN/ CD209 are C-type lectin receptors that are found on both macrophages and DCs $(11,28)$, so it was not surprising that there was expression of both lectin receptors on both FXIIIA ${ }^{+}$ and CD11 $\mathrm{c}^{+}$cells in normal human skin.

To evaluate the function of these 2 dominant dermal populations, we used 2 approaches. By comparing BDCA-1 and CD163 cells selected from collagenase digests of normal skin as inducers of an allogeneic MLR, we found that BDCA-1 ${ }^{+}$ cells were the main immunostimulatory population. These BDCA- $1^{+}$cells induced increased $\mathrm{T}$ cell proliferation when cultured before setting up the MLR, but were not as immunostimulatory as in vitro-matured DCs, suggesting that there were few mature DCs in normal skin compared with skin under inflammatory conditions such as psoriasis. This result reflects studies by Nestle et al. showing that bulk tissue émigrés from normal skin are not as stimulatory as those from patients with psoriasis (20). In comparison, CD163 ${ }^{\text {hi }}$ cells were not immunostimulatory in an allo-MLR, nor were they induced to be stimulatory, although they may possess some antigen-presenting capacity with upregulation of MHC class II molecules (29).

Skin tattoos provided a second functional study: the phagocytic activity of tissue macrophages. Pigment granules were found in lysosomal-like cellular structures, and cells containing pigment stained uniformly with CD163. The fate of tattoo pigment injected into dermal tissues has been studied in the past, and fibroblasts were considered to be the primary long-term reservoir of the pigment granules, with pigment in occasional macrophages $(30,31)$. However, our data suggest that macrophages are indeed a significant store of the dermal pigment. The cells with pigment were 

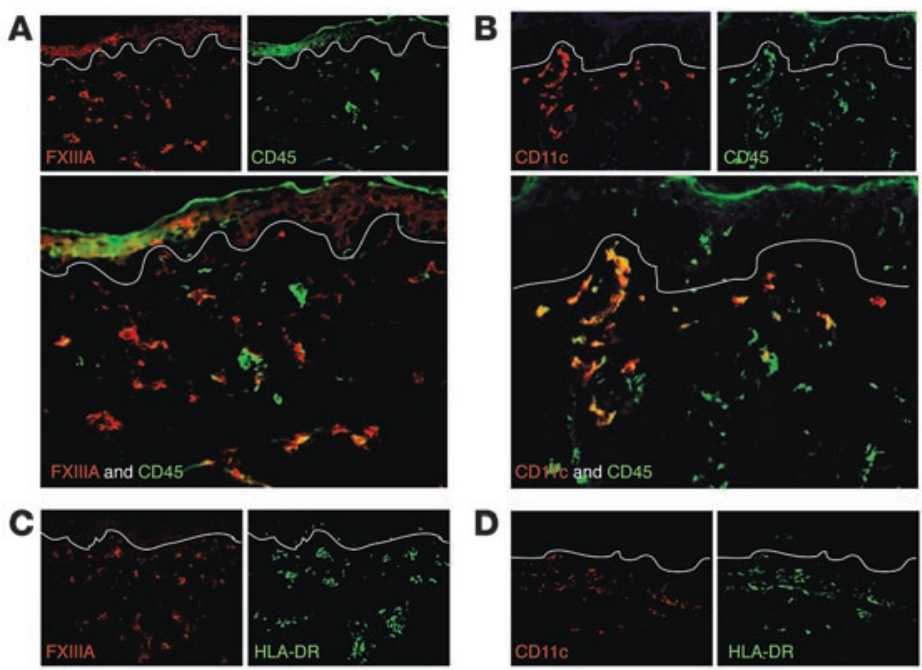

D
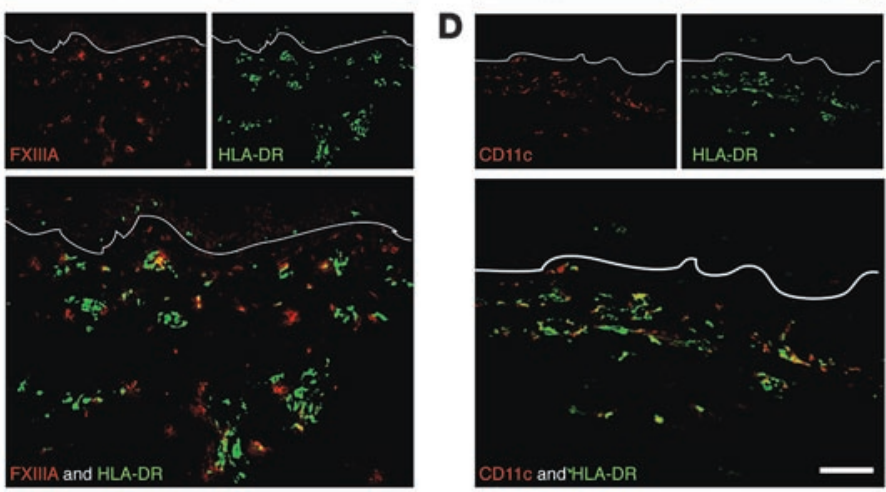

Figure 5

HLA-DR and CD45 mark both CD11 $\mathrm{c}^{+}$and FXIIIA+ cells. FXIIIA ${ }^{+}$cells had a lower expression level of CD45 (A) than did CD11 $\mathrm{c}^{+}$cells (B). HLA-DR was also expressed to a lower extent on $\mathrm{FXIIIA}^{+}$cells $(\mathbf{C})$ than on $\mathrm{CD} 11 \mathrm{C}^{+}$cells (D). Scale bar: $100 \mu \mathrm{m}$.

$\mathrm{CD}_{163^{+}}$and BDCA-1-, were round, had numerous microvillous projections, and the pigment was contained within membranebound structures (lysosomes). These characteristics are consistent with macrophages $(31,32)$.

In summary, we have identified $\mathrm{CD} 11 \mathrm{c}^{+}$cells as immature myeloid DCs in normal human skin and FXIIIA ${ }^{+}$cells as tissue-resident macrophages, not DCs, as they were previously classified. In future stud- ies of cutaneous DCs, we recommend considering the use of CD11c/BDCA-1 and CD163 as alternative markers to identify dermal DCs and macrophages, respectively, because they are more specific and more useful in flow cytometry applications.

\section{Methods}

Skin samples. Skin punch biopsies (6 $\mathrm{mm}$ diameter) were obtained from 15 normal volunteers under a protocol approved by the Rockefeller University Institutional Review Board. Informed consent was obtained. Biopsies were frozen in OTC (Sakura) and stored at $-80^{\circ} \mathrm{C}$ for immunohistochemistry and immunofluorescence. Normal samples from abdominoplasty were processed within 4 hours after surgery. These abdominoplasty samples were also the source of tattoo material $(n=2)$.

Immunohistochemistry. Normal skin sections $(n=15)$ were stained with purified mouse anti-human $\mathrm{CD} 11 \mathrm{c}$ (diluted 1:100) and sheep affinity-purified anti-human FXIIIA (diluted 1:100; Enzyme Research Laboratories). Biotin-labeled horse anti-mouse and biotinlabeled rabbit anti-sheep antibodies (Vector Laboratories) were used to detect CD11c and FXIIIA antibodies, respectively. The staining signal was amplified with avidin-biotin complex (Vector Laboratories) and developed using chromogen 3-amino-9-ethylcarbazole (Sigma-Aldrich). The number of positive cells per mm was counted manually using computer-assisted image analysis (NIH Image 6.1; http://rsb.info.nih.gov/nih-image). For FXIIIA immunostaining, several additional antibodies were screened, including AC-1A1 (Labvision), E5014 (Spring Bioscience), and E980.1 (Biogeniex). We chose the sheep affinity-purified antibody because it gave the most specific cellular staining with the least background epidermal staining.

Antibodies. All antibodies used for immunofluorescence and FACS of normal tissue are listed in Supplemental Tables 1 and 2.

Immunofluorescence. Normal skin sections $(n=6)$ were fixed with acetone and blocked in 10\% normal goat serum (Vector Laboratories) for 30 minutes. Primary antibodies CD11c and FXIIIA were incubated overnight at $4{ }^{\circ} \mathrm{C}$ and amplified with the appropriate secondary antibody: goat anti-mouse IgG1 conjugated with Alexa Fluor 488 or 568, or donkey anti-sheep conjugated with Alexa Fluor 488 or 568, respectively. For colocalization with CD11c or FXIIIA, sections were then costained overnight with a second antibody, as listed in Supplemental Table 1, and amplified with the appropriate goat-anti mouse secondary antibody

\section{Figure 6}

Cutaneous DCs compared with blood DCs. (A) There were 3 nonoverlapping DC populations in peripheral blood, gating on $\mathrm{Lin}^{-} \mathrm{CD} 11 \mathrm{C}^{+} \mathrm{HLA}-\mathrm{DR}^{+}$cells: BDCA $-1^{+}, \mathrm{CD}_{16}^{\mathrm{hi}}$, and BDCA-3+. (B) Blood BDCA-1+ (left) and BDCA-3+ (right) cells were both HLA-DR+CD14(C) In dermal single-cell suspensions, BDCA-1+ cells acquired CD16. (D) BDCA-1+ (left) dermal cells increased HLA-DR expression, and BDCA-3+ (right) dermal cells acquired low-level CD14 expression.
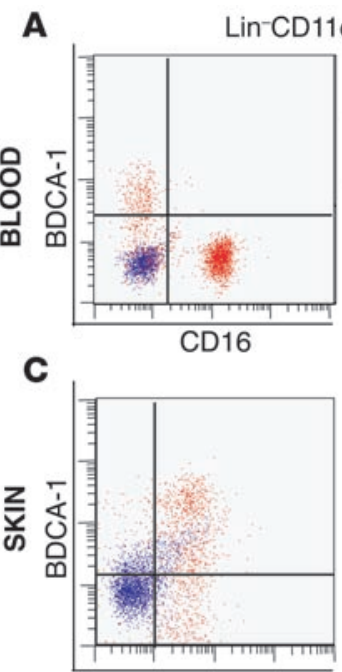

CD16
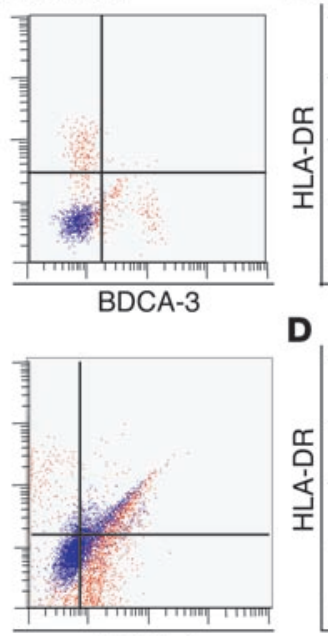

BDCA-3

D
B $\quad$ BDCA-1+
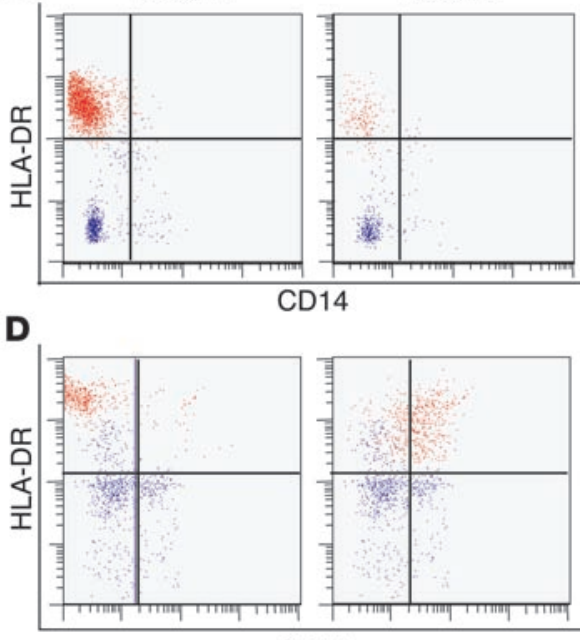

BDCA-3+

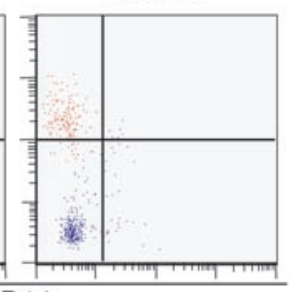

CD14

CD14 
A

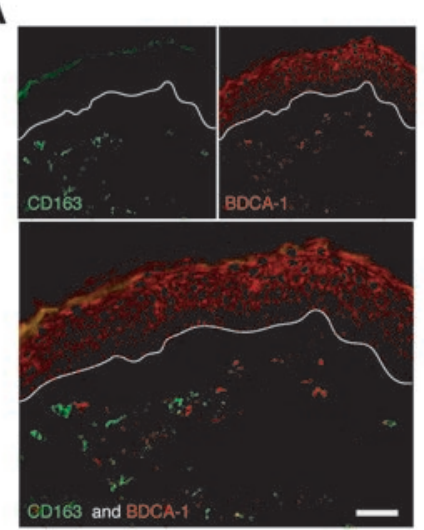

B

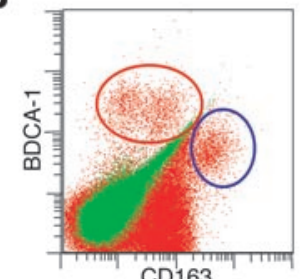

CD163
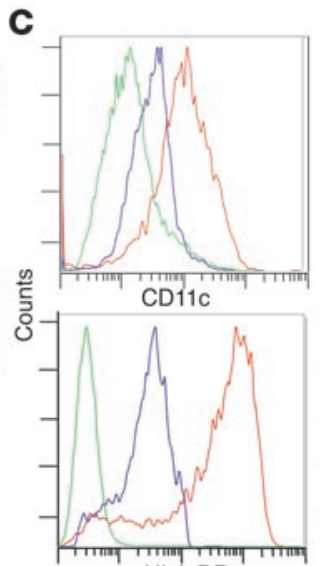

D

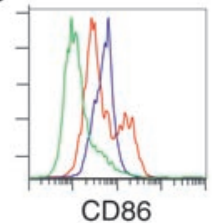

CD86
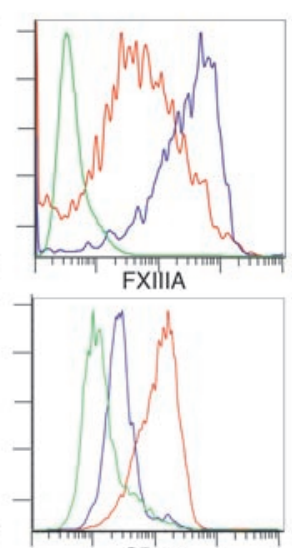

CD45
Figure 7

BDCA-1 and CD163 are alternative markers for CD11C and FXIIIA, respectively. (A) BDCA-1 and CD163 identified discrete populations of dermal cells. (B) BDCA-1+ cells (red circle) and CD163+ cells (blue circle) were also discrete populations in dermal single-cell suspensions. (C) FACS histograms gated on BDCA-1+ cells (red line), CD163+ cells (blue line), or isotype (green line). BDCA-1+ cells were CD11 $\mathrm{c}^{\text {hi }}$, FXIIIA $A^{\mathrm{lo}}, \mathrm{HLA}-\mathrm{DR} \mathrm{R}^{\mathrm{hi}}$, and CD45 ${ }^{\mathrm{hi}}$. CD163 ${ }^{+}$cells were CD11 $\mathrm{c}^{\text {mid }}$, FXIIIA ${ }^{\text {hi }}$, HLA-DR ${ }^{\text {mid }}$, and CD45 ${ }^{\circ}$. (D) A subset of BDCA-1+ cells was CD86 ${ }^{\mathrm{hi}}, \mathrm{CD} 83^{+}$, and DC-LAMPhi. Representative graphs from 3 experiments. Scale bar: $100 \mu \mathrm{m}$.
(Supplemental Table 1). Images were acquired using appropriate filters of a Zeiss Axioplan 2I microscope with Plan Apochromat $20 \times 0.7$ numerical aperture lens and a Hagamatsu orca ER-cooled charge-coupled device camera, controlled by METAVUE software (Universal Imaging). Dermal collagen fibers gave green autofluorescence. Antibodies conjugated with a fluorochrome often gave background epidermal fluorescence. Images in each figure are presented as single-color stains of green and red to make apparent the localization of 2 markers on similar or different cells. Merged images are shown below the single-color stains. Cells that coexpress the 2 markers in a similar location are often yellow in color.

\section{Figure 8}

BDCA-1+ cells are more immunostimulatory. (A) Postsort dot plot of dermal cells from normal skin into BDCA-1+ and $\mathrm{CD}_{163^{+}}$populations (red, left and right panels, respectively) compared with isotype (blue). (B) Positive control (monocyte-derived mature DC) for MLR on day 8 after sorting at a 1:100 stimulator/responder ratio. Gate contains $\mathrm{CD}^{+}$proliferating T cells with left-shifted CFSE. (C) Using BDCA-1+ sorted cells as stimulators (1:10 ratio), $9.1 \%$ of the $T$ cells proliferated; using $\mathrm{CD}^{163^{+}}$cells (1:10 ratio), $2.1 \%$ of live T cells proliferated. Background proliferation of $\mathrm{T}$ cells alone without stimulation was $1.0 \%$. (D) After cells had been sorted and cultured for 2 days with cytokines to induce maturation, there was a marked increase in the T cell stimulatory capacity of BDCA- $1^{+}$cells $(25.2 \%, 1: 100$ ratio) versus CD163 (2.2\%, 1:250 ratio). Representative graphs from 3 experiments.
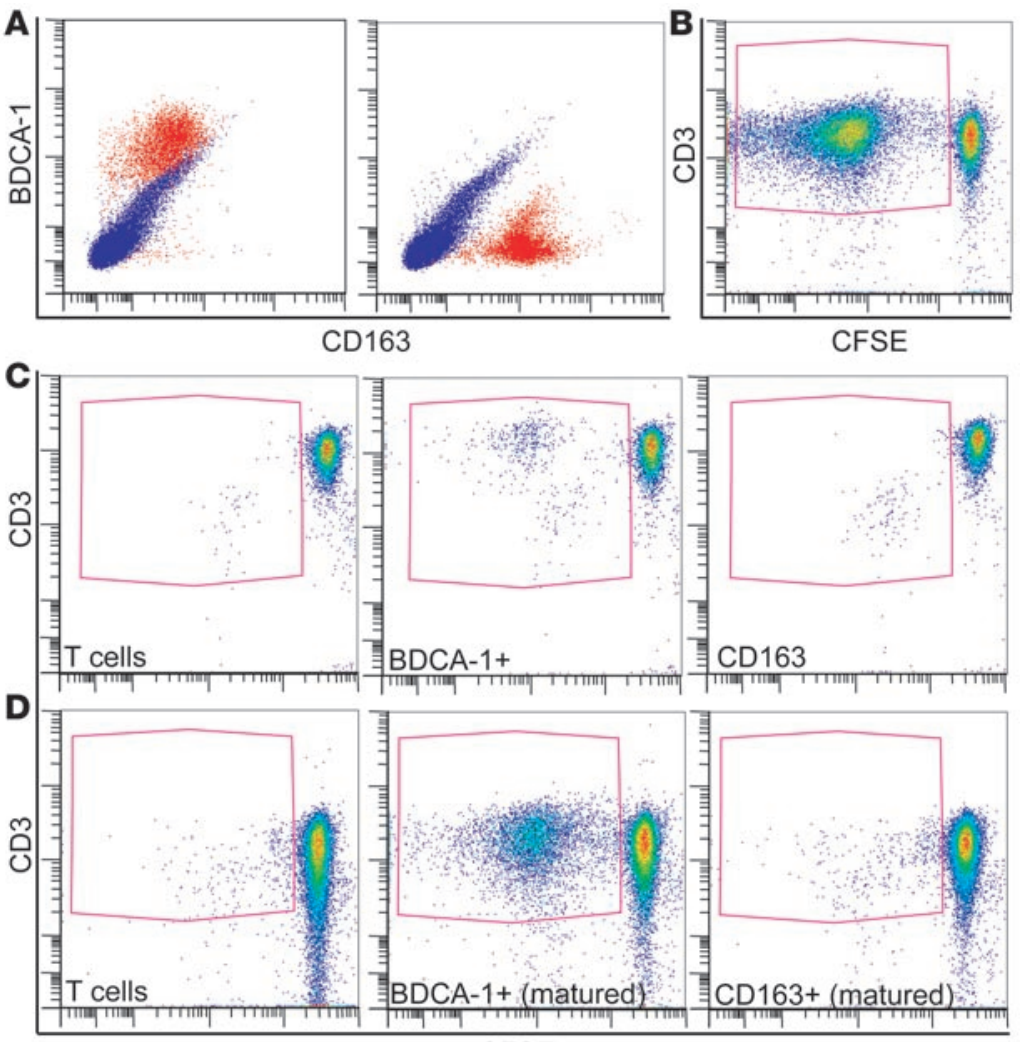

CFSE 

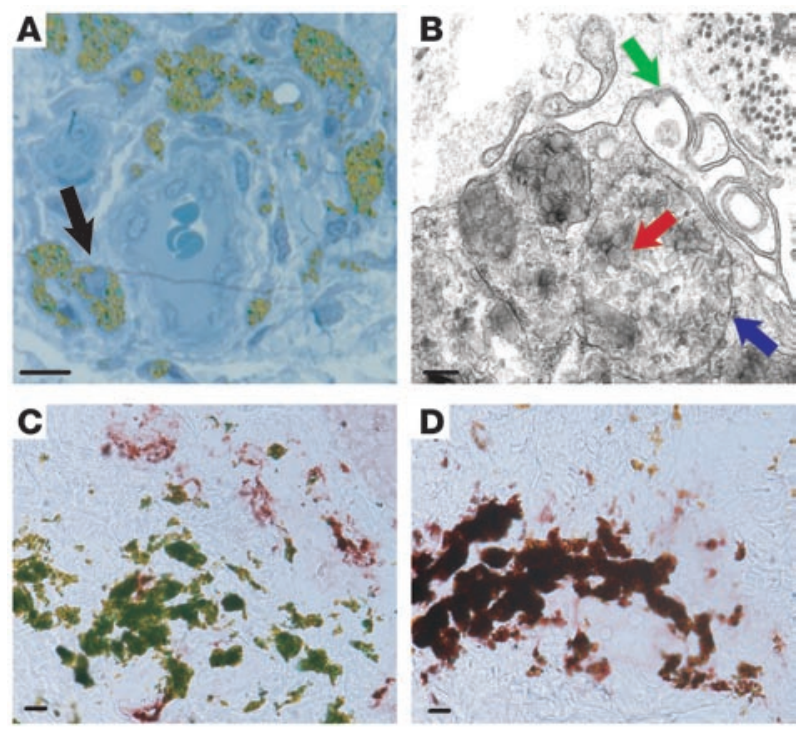

Electron microscopy. Skin was fixed in $2.5 \%$ glutaraldehyde and processed by routine transmission electron microscopy procedure. Semithin plastic sections were stained with toluidine blue for light microscopic evaluation. Ultrathin (65-nm) sections were cut with a diamond knife, collected on copper grids, and stained with both uranyl acetate and lead citrate before viewing in a Tecnaispirit electron microscope (FEI Company) equipped with an Ultrascan 895 charge-coupled device camera (Gatan).

Skin sample processing. Dermal single-cell suspensions from normal skin were obtained using a modified collagenase/dispase method (16). Subcutaneous fat was excised, and remaining tissue was washed with PBS. The dermal layer was heavily scored with a scalpel and incubated in $1 \mathrm{mg} / \mathrm{ml}$ type 1 collagenase (Invitrogen), $1 \mathrm{mg} / \mathrm{ml}$ dispase (Invitrogen), and $1 \%$ penicillin-streptomycin solution (Sigma-Aldrich) overnight at $37^{\circ} \mathrm{C}$. The epidermis was peeled off and discarded, and the dermis was transferred to fresh RPMI 1640 supplemented with $10 \%$ pooled human serum (Mediatech Inc.), 0.1\% gentamicin reagent solution (Invitrogen), and 1\% 1 M HEPES buffer (Sigma-Aldrich). The dermis was incubated 24-48 hours at $37^{\circ} \mathrm{C}$, and the supernatant was collected and filtered with $40-\mu \mathrm{m}$ cell strainers (BD Biosciences). Cells were then either used immediately (for MLR; $n=3$ ) or frozen in RPMI 1640 (Invitrogen) and 10\% DMSO (ATCC) for FACS $(n=3)$.

FACS. Cells were stained with the antibodies listed in Supplemental Table 2. Briefly, cells were stained for 20 minutes at $4{ }^{\circ} \mathrm{C}$, washed with FACSwash (PBS $0.1 \%$ sodium azide and 2\% FBS), and resuspended in $1.3 \%$ formaldehyde (Fisher Scientific) in FACSwash. To detect DC-LAMP, cells were first stained for surface markers, then permeabilized (FacsPerm; BD) before intracellular staining. Samples were acquired using FACSCanto or LSR-II (both from BD Biosciences) and analyzed with FlowJo (Treestar). Appropriate isotypes were used.

FACS and MLR. Dermal cells from single-cell suspensions of normal skin were stained with BDCA-1, CD19, and CD163 antibody and sorted on a FACSAria (BD Biosciences) using a low-pressure setting. Two populations were obtained: $\mathrm{BDCA}-1^{+} \mathrm{CD} 19^{-}$and $\mathrm{CD} 163^{+}$. A post-sort collection was performed to confirm the purity of each stimulating population. For some experiments, sorted cells were cultured for 2 days with and without cytokines for maturing DCs ex-vivo (IL-1 $\beta$, IL-6, TNF- $\alpha$, and PGE 2 ), then washed and prepared for the MLR.

Responding T cells were obtained from a normal volunteer by density centrifugation over Ficoll-Paque Plus (Amersham Biosciences), followed

\section{Figure 9}

CD163 ${ }^{+}$cells phagocytose large particles and have the structural features of macrophages. (A) Tattoo skin section $(0.5 \mu \mathrm{m})$ stained with toludine blue. Cells containing green tattoo dye in their cytoplasm (black arrow) surrounded a blood vessel. (B) Electron microscopy of a tattoo showed that dye particles (red arrow) were membrane bound (blue arrow) within the cytoplasm of a cell with multiple microvillus protrusions (green arrow). (C and D) Cells containing green tattoo dye particles stained for CD163 (D) but not BDCA-1 (C). Scale bar: $10 \mu \mathrm{m}$ (A, C, and D); $200 \mathrm{~nm}$ (B). by $\mathrm{T}$ cell purification using a $\mathrm{T}$ cell-negative selection kit (Dynal). T cells were labeled with $10 \mu \mathrm{m}$ CFSE and cocultured with either BDCA- $1^{+}$or CD163 ${ }^{+}$sorted cells at ratios of 1:10, 1:50, and 1:100 (depending on cell yield). $T$ cells without a stimulator population were used as a negative control, and monocyte-derived mature DCs were added to T cells as a positive control. The process for making mature DCs was previously described (33). T cell proliferation was analyzed on day 8 after sorting. The cultures were harvested, stained with $250 \mathrm{ng} / \mathrm{ml}$ propidium iodide (PI) to label dead cells, and CD3-APC (BD) for 15 minutes at room temperature. PI-negative cells were gated and then plotted as CFSE versus $\mathrm{CD}^{+}$cells, where proliferating cells diluted their content of CFSE and moved to the left of the nonproliferating cells. The CFSE-low cells were quantified as a percentage of live cells in the culture (34).

Statistics. A 2-tailed paired Student's $t$ test was used to compare CD11c and FXIIIA $^{+}$cells in normal skin sections. Results are shown as mean $\pm \mathrm{SEM}$. A $P$ value less than 0.05 was considered significant.

\section{Acknowledgments}

This research was supported by NIH grants R01 AI-49572, AI-49832, UL1 RR024143, and AI40045 (R.M. Steinman). M.A. Lowes is supported by NIH grant 1 K23 AR052404-01A1, and L. Zaba is supported by NIH MSTP grant GM07739. We thank plastic surgeons A.N. LaBruna and D.M. Senderoff for their generous donation of abdominoplasty surgical waste, $\mathrm{H}$. Shio and A. Khatcherian for their technical assistance on electron microscopy and immunohistochemistry of the tattoo, respectively, and A. Piperno for the kind gift of monoclonal antibody DEC-205/CD205. We also appreciate the assistance and advice of the Flow Cytometry Core Facility (S. Mazel) and Bio-imaging Resource Center (A. North) at Rockefeller University. We thank Patricia Gilleaudea and Mary Whalen-Sullivan for excellent care of our patients.

Received for publication March 7, 2007, and accepted in revised form June 6, 2007.

Address correspondence to: Michelle A. Lowes, Laboratory for Investigative Dermatology, The Rockefeller University, 1230 York Avenue, New York, New York 10021, USA. Phone: (212) 327-7576; Fax: (212) 327-8353; E-mail: lowesm@rockefeller.edu. 
1. Larregina, A.T., and Falo, L.D. 2005. Changing paradigms in cutaneous immunology: adapting with dendritic cells. J. Invest. Dermatol. 124:1-12.

2. Valladeau, J., and Saeland, S. 2005. Cutaneous dendritic cells. Semin. Immunol. 17:273-283.

3. Hunger, R.E., et al. 2004. Langerhans cells utilize CD1a and langerin to efficiently present nonpeptide antigens to T cells. J. Clin. Invest. 113:701-708. doi:10.1172/JCI200419655.

4. Cerio, R., Griffiths, C.E., Cooper, K.D., Nickoloff, B.J., and Headington, J.T. 1989. Characterization of factor XIIIa positive dermal dendritic cells in normal and inflamed skin. Br. J. Dermatol. 121:421-431.

5. Henriksson, P., Becker, S., Lynch, G., and McDonagh, J. 1985. Identification of intracellular factor XIII in human monocytes and macrophages. J. Clin. Invest. 76:528-534.

6. Torocsik, D., Bardos, H., Nagy, L., and Adany, R. 2005. Identification of factor XIII-A as a marker of alternative macrophage activation. Cell. Mol. Life Sci. 62:2132-2139.

7. Freudenthal, P.S., and Steinman, R.M. 1990. The distinct surface of human blood dendritic cells, as observed after an improved isolation method. Proc. Natl. Acad. Sci. U. S. A. 87:7698-7702.

8. Summers, K.L., Hock, B.D., McKenzie, J.L., and Hart, D.N. 2001. Phenotypic characterization of five dendritic cell subsets in human tonsils. Am.J. Pathol. 159:285-295.

9. MacDonald, K.P., et al. 2002. Characterization of human blood dendritic cell subsets. Blood. 100:4512-4520.

10. Brigl, M., and Brenner, M.B. 2004. CD1: antigen presentation and T cell function. Annu. Rev. Immunol. 22:817-890.

11. Granelli-Piperno, A., et al. 2005. Dendritic cell-specific intercellular adhesion molecule 3 -grabbing nonintegrin/CD209 is abundant on macrophages in the normal human lymph node and is not required for dendritic cell stimulation of the mixed leukocyte reaction. J. Immunol. 175:4265-4273.

12. Krutzik, S.R., et al. 2005. TLR activation triggers the rapid differentiation of monocytes into macrophages and dendritic cells. Nat. Med. 11:653-660.

13. Gordon, S., and Taylor, P.R. 2005. Monocyte and macrophage heterogeneity. Nat. Rev. Immunol.
5:953-964.

14. Wang, F., et al. 2006. Prominent production of IL-20 by CD68+/CD11c+ myeloid-derived cells in psoriasis: gene regulation and cellular effects. J. Invest. Dermatol. 126:1590-1599.

15. Tormey, V.J., et al. 1997. T-cell cytokines may control the balance of functionally distinct macrophage populations. Immunology. 90:463-469.

16. Angel, C.E., et al. 2006. Cutting edge: CD1a+ antigen-presenting cells in human dermis respond rapidly to CCR7 ligands. J. Immunol. 176:5730-5734.

17. Nestle, F.O., Zheng, X.G., Thompson, C.B., Turka, L.A., and Nickoloff, B.J. 1993. Characterization of dermal dendritic cells obtained from normal human skin reveals phenotypic and functionally distinctive subsets. J. Immunol. 151:6535-6545.

18. Meunier, L., Gonzalez-Ramos, A., and Cooper, K.D. 1993. Heterogeneous populations of class II MHC+ cells in human dermal cell suspensions. Identification of a small subset responsible for potent dermal antigen-presenting cell activity with features analogous to Langerhans cells. J. Immunol. 151:4067-4080.

19. Deguchi, M., Aiba, S., Ohtani, H., Nagura, H., and Tagami, H. 2002. Comparison of the distribution and numbers of antigen-presenting cells among T-lymphocyte-mediated dermatoses: CD1a+, factor XIIIa+, and CD68+ cells in eczematous dermatitis, psoriasis, lichen planus and graft-versus-host disease. Arch. Dermatol. Res. 294:297-302.

20. Nestle, F.O., Turka, L.A., and Nickoloff, B.J. 1994. Characterization of dermal dendritic cells in psoriasis. Autostimulation of $\mathrm{T}$ lymphocytes and induction of Th1 type cytokines. J. Clin. Invest. 94:202-209.

21. Dupasquier, M., Stoitzner, P., van Oudenaren, A., Romani, N., and Leenen, P.J. 2004. Macrophages and dendritic cells constitute a major subpopulation of cells in the mouse dermis. J. Invest. Dermatol. 123:876-879.

22. Narbutt, J., et al. 2006. The number and distribution of blood dendritic cells in the epidermis and dermis of healthy human subjects. Folia Histochem. Cytobiol. 44:61-63.

23. Fivenson, D.P., and Nickoloff, B.J. 1995. Distinctive dendritic cell subsets expressing factor XIIIa,
CD1a, CD1b and CD1c in mycosis fungoides and psoriasis. J. Cutan. Pathol. 22:223-228.

24. de Saint-Vis, B., et al. 1998. A novel lysosome-associated membrane glycoprotein, DC-LAMP, induced upon DC maturation, is transiently expressed in MHC class II compartment. Immunity. 9:325-336.

25. Ebner, S., et al. 2004. Expression of C-type lectin receptors by subsets of dendritic cells in human skin. Int. Immunol. 16:877-887.

26. Fabriek, B.O., Dijkstra, C.D., and van den Berg, T.K. 2005. The macrophage scavenger receptor CD163. Immunobiology. 210:153-160.

27. Janeway, C., Travers, P., Walport, M., and Shlomchik, M. 2004. Immunobiology: the immune system in health and disease. 6th edition. Garland Science. New York, New York, USA. 800 pp.

28. McGreal, E.P., Miller, J.L., and Gordon, S. 2005. Ligand recognition by antigen-presenting cell C-type lectin receptors. Curr. Opin. Immunol. 17:18-24.

29. Steinman, R.M., and Hemmi, H. 2006. Dendritic cells: translating innate to adaptive immunity. Curr. Top. Microbiol. Immunol. 311:17-58.

30. Lea, P.J., and Pawlowski, A. 1987. Human tattoo. Electron microscopic assessment of epidermis, epidermal-dermal junction, and dermis. Int. J. Dermatol. 26:453-458.

31. Fujita, H., Nishii, Y., Yamashita, K., Kawamata, S., and Yoshikawa, K. 1988. The uptake and long-term storage of India ink particles and latex beads by fibroblasts in the dermis and subcutis of mice, with special regard to the non-inflammatory defense reaction by fibroblasts. Arch. Histol. Cytol. 51:285-294.

32. Taylor, C.R., Anderson, R.R., Gange, R.W., Michaud, N.A., and Flotte, T.J. 1991. Light and electron microscopic analysis of tattoos treated by Q-switched ruby laser. J. Invest. Dermatol. 97:131-136.

33. Lee, A.W., et al. 2002. A clinical grade cocktail of cytokines and PGE2 results in uniform maturation of human monocyte-derived dendritic cells: implications for immunotherapy. Vaccine. 20(Suppl. 4):A8-A22.

34. Parish, C.R., and Warren, H.S. 2001. Use of the intracellular fluorescent dye CFSE to monitor lymphocyte migration and proliferation. In Current protocols in immunology. John Wiley \& Sons Inc. Hoboken, New Jersey, USA. Unit 4.9.1. 